Method IFX trough levels and ATI were measured in patients attending for IFX infusions from May 2016 at a tertiary referral centre. IFX and ATI trough levels were measured using drug tolerant enzyme-linked immunosorbent assays (Immundiagnostik, UK). Positive ATI were defined as levels $>10 \mathrm{mg} / \mathrm{L}$.

Treatment failure was defined as ongoing/worsening of symptoms attributable to IBD, requiring alteration in treatment. Fisher's exact test was used to compare rates of previous IFX exposure, concomitant immunosuppression (IS) and treatment failure between those with positive and negative ATI.

Result TDM at dose 4 (week 14) was available from 50 patients $(25$ Crohn's disease, 24 ulcerative colitis and 1 IBDunclassified) and is shown in table 1. 14/50 (28\%) were positive for ATI. In the 10 patients that had subsequent TDM after ATI was noted to be positive, 9 remained positive- sustained ATI and 1 became negative- transient ATI. There was no significant difference in use of concomitant IS between those with positive and negative ATI. However, those with positive ATI were more likely to have received previous IFX $(5 / 14$ compared to $1 / 36, \mathrm{p}<0.01)$. Of the 9 patients who developed positive ATI with no previous IFX exposure, 4 were not receiving concomitant IS.

Only $8 / 50(16 \%)$ patients had a therapeutic IFX trough levels (range 3.2-21.4 mg/L); all negative for ATI. Median IFX level was significantly lower in those with positive ATI compared to those who were negative $(0.9 \mathrm{mg} / \mathrm{L}$ v $1.7 \mathrm{mg} / \mathrm{L} ; \mathrm{p}=0.02)$.

Of those with positive ATI at first maintenance dose, 10/14 $(71 \%)$ were treatment failures by second maintenance dose, compared to $10 / 36(28 \%)$ in those with negative ATI $(\mathrm{p}<0.01)$.

\begin{tabular}{|c|c|c|c|c|c|}
\hline \multirow{2}{*}{$\begin{array}{l}\text { ATI } \\
\text { status }\end{array}$} & \multicolumn{5}{|c|}{ IFX trough level $(\mathrm{mg} / \mathrm{L})$} \\
\hline & $\begin{array}{l}\text { Undetectable } \\
(<0.8)\end{array}$ & $\begin{array}{l}\text { Sub-therapeutic } \\
(0.8-2.9)\end{array}$ & $\begin{array}{l}\text { Therapeutic } \\
(3-7)\end{array}$ & $\begin{array}{l}\text { Supra- } \\
\text { therapeutic } \\
(>7)\end{array}$ & Total \\
\hline Positive & 6 & 8 & 0 & 0 & 14 \\
\hline Negative & 7 & 20 & 8 & 1 & 36 \\
\hline Total (\%) & $13(26)$ & $28(56)$ & $8(16)$ & $1(2)$ & 50 \\
\hline
\end{tabular}

Conclusion At first maintenance dose, nearly a third of patients had developed positive ATI. These patients have lower IFX levels and a higher risk of treatment failure by the second maintenance dose. Therefore, TDM following induction therapy may be important in identifying patients who require rapid treatment alteration.

\section{PWE-066 MICRORNA SIGNATURES CAN DIFFERENTIATE BETWEEN INFLAMED AND NON INFLAMED COLONIC MUCOSA OF ULCERATIVE COLITIS PATIENTS}

Swati Valmiki*. Jawaharlal Nehru University, New Delhi, India

\subsection{6/gutjnl-2018-BSGAbstracts. 198}

Introduction Ulcerative colitis (UC) is a form of inflammatory bowel diseases (IBD) which are chronic, relapsing and idiopathic disorders of gastrointestinal tract. The aetiology of IBD has not been defined yet but a complex interplay of microbial, genetic and environmental factors is known to play role in its pathogenesis. Recently, microRNAs have emerged as an epigenetic regulator of inflammation during IBD. MicroRNA target the genes involved in major signalling pathways and regulate there expression thereby affecting the overall inflammatory pathway. In this study we looked at the miRNA profile of inflamed and non inflamed regions of UC patients. We also studied the biological relevance of this dysregulated expression by looking at the potential targets of these miRNA and the biological pathways involved.

Methods To investigate the expression profile of miRNAs we collected the colonic mucosal biopsies from the endoscopically inflamed and non inflamed regions of UC patients. Colonic biopsies were also collected from control individuals. The differential expression of miRNA was studied by microarray and qRT-PCR. The potential targets of miRNA were predicted using bioinformatics tools such as TargetScan, MIRDB, DIANA-MicroT, microrna.org and Pictar. The pathways involved were identified by mirPath v.3:DIANA TOOLS.

Results The miRNA profile of inflamed colonic mucosa was found to be significantly different from the non inflamed one in microarray. The real time analysis showed a significant upregulation in the expression of miR-125b and miR-223 in the inflamed colonic mucosa as compared to non inflamed mucosa. TRAF6 which is a potential target of miR-125b and an important signalling molecule of NFkB pathway, showed a significant downregulation in UC patients as compared to controls. Similarly, IKK alpha which is targeted by miR-223 showed a significant downregulation in its expression in UC patients.

Conclusions Our study indicate towards the spatial expression of miRNA during UC and their biological relevance. MiR-223 showed a disease independent behaviour therefore, it could be developed as a biomarker for UC. Studying these miRNA and the signalling pathways in detail, could provide better insight of disease pathogenesis and provide scope for their use in therapeutics.

\section{PWE-067 HEALTHCARE RESOURCE UTILISATION AND QOL IN PATIENTS WITH UC BY DISEASE SEVERITY: ICONIC BASELINE DATA}

${ }^{1}$ Stijn van Haaren*, ${ }^{2}$ Subrata Ghosh, ${ }^{3}$ Laurent Peyrin-Biroulet, ${ }^{4}$ Francesc Casellas, ${ }^{1}$ Ciara O'Shea, 'Wan-Ju Lee, ${ }^{1}$ Brandee Pappalardo, ${ }^{1}$ Joel Petersson. ${ }^{1}$ Abbvie, North Chicago, UK; ${ }^{2}$ College of Medical and Dental Sciences University of Birmingham, Birmingham, UK; ${ }^{3}$ Nancy University Hospital, Nancy, France; ${ }^{4}$ Hospital Universitari Vall d'Hebron, Barcelona, Spain

\subsection{6/gutjnl-2018-BSGAbstracts. 199}

Introduction ICONIC is a prospective, multi-country $(n=33)$ observational study, assessing cumulative burden in adult ulcerative colitis (UC) patients (pts) under routine care. Assessments of healthcare resource utilisation (HCRU), disease severity and impact on quality of life will be captured at 6 month intervals through 2 years. This analysis evaluated baseline (BL) HCRU, work productivity, and quality of life among UC pts with different level of disease severity.

Methods Pts with early UC (diagnosed $\leq 36$ months) were enrolled irrespective of disease severity or treatment. For this analysis, we evaluated pts stratified by physician assessment of disease severity into severe, moderate, mild and remission groups. BL characteristics described: Simple Clinical Colitis Activity Index (SCCAI), UC-related HCRU measured during the 6 months prior to study enrollment (i.e., visits to treating 
physicians or other IBD-associated healthcare professionals, emergency room (ER) visits, hospitalizations and hospital admissions for surgeries); Health Related Quality of Life (HRQoL), pt-reported employment/UC-related sick leave status and Work and Productivity Activity Index (WPAI:GH; including work time missed (absenteeism), impairment while working (presenteeism), overall work productivity impairment, and daily activity impairment domains).

Results A total of 1816 UC pts were enrolled; mean \pm SD age was $38.5 \pm 14.6$ years and $833(45.9 \%)$ were female. At BL, 230 pts $(12.7 \%)$ were in remission, 672 pts $(37.0 \%)$ had mild UC, 668 pts $(36.8 \%)$ had moderate UC, and $234(12.9 \%)$ had severe UC. Compared to pts in remission, pts with moderate to severe UC had 1.8 to 2.6-fold higher rates of hospitalizations and 1.6 to 2.5 -fold higher rates of ER visits over the past 6 months. Pt with moderate and severe disease were associated with lower SIBDQ scores and higher WPAI:GH domain scores (i.e., greater impairment on work productivity) than pts with mild disease or those in remission.

$172(9.5 \%)$ pts reported to be unemployed at BL. 183 $(10.1 \%)$ reported sick leave at BL. Sick leave time ranged from $<2$ months (59.6\%), 2-4 months (12.6\%), >4 months (22.4\%).

Conclusion The direct and indirect burden of UC is substantial, as measured by healthcare resource utilisation and worklife impact. Pts with moderate and severe UC not only were associated with higher rates of urgent care in hospitalisation and ER visits, but also with poorer quality of life, higher unemployment, sick leave and impaired work productivity than pts with mild UC or in remission. For pts with mild UC, despite comparable HCRU to pts in remission, increased impact on work productivity remains a concern.

\section{PWE-068 ADVERSE EVENTS IN ELDERLY INFLAMMATORY BOWEL DISEASE PATIENTS MANAGED WITH ANTI-TNF THERAPY}

Conchubhair Winters*, Peter Mooney. Leeds Teaching Hospitals Trust, Leeds, UK

10.1136/gutjnl-2018-BSGAbstracts.200

Introduction In a population with an increasing life expectancy, a sizable proportion of inflammatory bowel disease (IBD) patients are elderly. The management of IBD often requires immunosuppressing anti-tumour necrosis factor (antiTNF) drugs which add to the immunosuppressive effects of ageing. Evidence for the safety of anti-TNF therapy in the elderly is scarce. Our objective was to assess the safety of antiTNF therapy in the elderly considering their co-morbidities and immunomodulators (IM).

\section{Methods}

Retrospective single centre study The IBD database of a large teaching hospital was interrogated for patients aged $>65$ years who had been prescribed infliximab or adalimumab. Patient electronic records were reviewed along with general practice prescribing records. Data was collected on co-morbidities, IM use, hospitalisations, significant adverse events (any reaction requiring discontinuation of the anti-TNF), and antibiotic prescriptions. Charlson Co-Morbidity index (CCI) was calculated. Results 80 patients (51 female) aged $>65$ received either infliximab $(n=50)$ or adalimumab $(n=30)$. Crohn's disease $(n=70)$ was more common and 34 patients were on a concomitant IM. The median duration of follow-up (FU) was 4 years and the median duration of therapy was 14 months. There were 5 deaths during FU, 4 after cessation of anti-TNF (2 pneumonias, 1 chronic obstructive pulmonary disease, 1 malignancy) and 1 patient was still on an anti-TNF (Crohn's related malnutrition). Seven patients developed cancer, 5 still on an anti-TNF and the other two were one and two years post-cessation of anti-TNF. Of the 5 patients who developed cancers on an anti-TNF, all 5 restarted their anti-TNF after treatment of the cancer. Eight patients (10.5\%) required hospitalisation due to what was felt to be an anti-TNF related event (7 infective, 1 allergic reaction). Patients on an IM had a $15.4 \%$ chance of anti-TNF related hospitalisation vs $4.4 \%$ in those not on a concomitant IM $(p=0.09)$. Concomitant IM use had no statistical impact on the risk of developing a cancer $(9.1 \%$ on an IM vs $6.5 \%$ not on an IM, p=0.49). Of those that required antibiotics, IM use did not seem to increase this risk $(p=0.43)$. Thirty one percent of those that stopped their anti-TNF $(n=50)$ did so because of an adverse event. When $\mathrm{CCI}=0$ was compared with a CCI $>0$, they were no more likely to still be on an anti-TNF after 12 months.

Conclusions In this series, we were unable to demonstrate a relationship between co-morbidities and tolerance of antiTNF therapy. There was, although not reaching statistical significance, a relationship between concomitant IMs and risk of hospitalisation due potential anti-TNF related events. Elderly patients are more likely to stop anti-TNFs than the younger populations used in larger trials. Concomitant IMs must be carefully considered to reduce the risk of adverse events.

\section{PWE-069 MEASURING THIOGUANINE NUCLEOTIDE (6-TGN) LEVELS AND CLINICAL RESPONSE IN IBD}

Mehmet Yalchin*, Lukasz Kamieniarz, Rigers Cama, Samuel Tribich, Andreas Koutsoumpas. Royal Free Hospital, London, UK

\subsection{6/gutjnl-2018-BSGAbstracts.201}

Introduction Monitoring levels of 6-TGN and titrating dose of Azathiopurine (AZA) and Mercaptopurine (6-MP) accordingly to achieve therapeutic concentrations of 6-TGN has been reported to improve outcomes in the treatment of Ulcerative colitis (UC) and Crohn's Disease (CD). The aim of our study was to show how levels of 6-TGN corresponds to clinical outcome.

Method This was a single centre (Royal Free Hospital), retrospective study, of patients receiving AZA or 6MP. We identified our patients by collating those who had been dispensed AZA or $6 \mathrm{MP}$ over the past 2 years. We were then able to access their electronic database, and record whether 6-TGN levels were subsequently taken to titrate treatment, and assess clinical outcome.

Results 109 from 426 patients have so far been analysed $(\mathrm{F}=54$ [50\%]). $\mathrm{UC}=45$ [41\%] $\mathrm{CD}=64$ [59\%].

Mean duration on thiopurines was 7.6 years. Mean disease duration was 16.2 years.

$74 / 109(68 \%)$ had 6-TGN levels at some stage.

42/74 (57\%) had therapeutic levels, whilst 32/74 (43\%) did not.

49/74 (66\%) were in clinical remission. 25/74 (34\%) were not. 


\title{
Correction: PWE-067 Healthcare resource utilisation and QOL in patients with UC by disease severity: iconic baseline data
}

\author{
van Haaren S, Ghosh S, Peyrin-Biroulet L, et al. PWE-067 Healthcare resource utilisation \\ and QOL in patients with UC by disease severity: iconic baseline data. Gut 2018;67:A100- \\ A101. doi: 10.1136/gutjnl-2018-BSGAbstracts.199. \\ The correct order of authors is: \\ Subrata Ghosh, Laurent Peyrin-Biroulet, Francesc Casellas, Ciara O’Shea, Wan-Ju Lee, Brandee \\ Pappalardo, Joel Petersson. \\ (c) Author(s) (or their employer(s)) 2018. No commercial re-use. See rights and permissions. Published by BMJ. \\ Gut 2018;67:2232. doi:10.1136/gutjnl-2018-BSGAbstracts.199corr1
}

D) Check for updates 